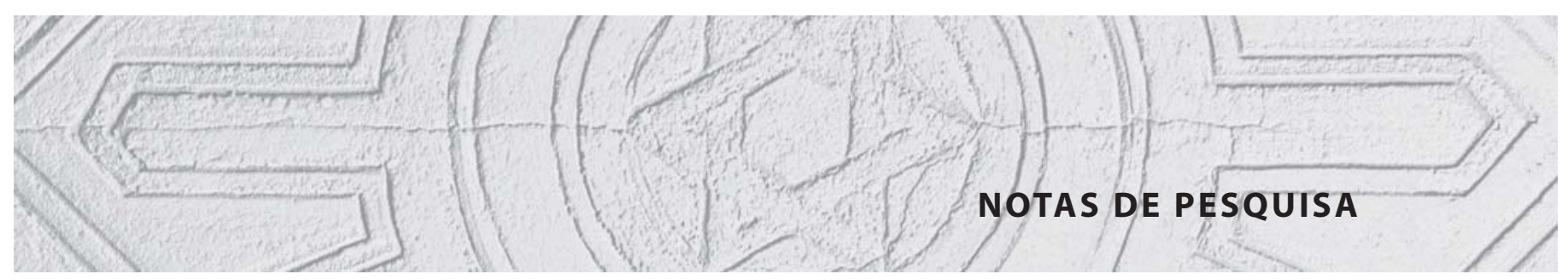

\title{
Coleção entomológica do Instituto Oswaldo Cruz: resgate de acervo científico-histórico disperso pelo Massacre de Manguinhos
}

\author{
The entomology collection at Instituto Oswaldo Cruz: restoring a \\ historical scientific collection scattered by the Manguinhos Massacre
}

\author{
Jane Costa \\ Pesquisadora e chefe/curadora da \\ Coleção Entomológica / Laboratório \\ de Biodiversidade Entomológica / \\ Instituto Oswaldo Cruz/ \\ Fundação Oswaldo Cruz \\ jcosta@ioc.fiocruz.br
}

\section{Danielle Cerri}

Assistente da curadoria da Coleção Entomológica / Laboratório de Biodiversidade Entomológica / Instituto Oswaldo Cruz /

Fundação Oswaldo Cruz

cerri@ioc.fiocruz.br

Instituto Oswaldo Cruz

Av. Brasil, 4365

21040-900 Rio de Janeiro -

RJ - Brasil

\section{Magali Romero de Sá}

Pesquisadora da Casa de Oswaldo Cruz / Fundação Oswaldo Cruz magali@coc.fiocruz.br

\section{Carlos José Einicker \\ Lamas}

Professor e curador da Coleção de Diptera / Museu de Zoologia / Universidade de São Paulo einicker@usp.br
COSTA, Jane et al. Coleção entomológica do Instituto Oswaldo Cruz: resgate de acervo científico-histórico disperso pelo Massacre de Manguinhos. História, Ciências, Saúde - Manguinhos, Rio de Janeiro, v.15, n.2, p.401-410, abr.-jun. 2008.

\section{Resumo}

Uma das mais ricas, antigas e diversificadas coleções entomológicas da América Latina, que alberga relevante parcela de nossa biodiversidade, foi drasticamente penalizada durante o episódio que ficou conhecido como o Massacre de Manguinhos, no qual dez eminentes pesquisadores tiveram seus direitos políticos cassados. Esta nota relata uma ínfima parcela da tragédia que desmantelou parte desse acervo e como 8.554 exemplares valiosos da ordem Diptera, pertencentes a 35 famílias, incluindo 99 exemplares-tipos, foram reincorporados à Coleção Entomológica do Instituto Oswaldo Cruz, em suas posições originais nos armários de aço, após 35 anos. Esta reincorporação representa o primeiro resgate científico-histórico desse acervo, após o período da ditadura militar no Brasil.

Palavras-chave: Coleção Entomológica do Instituto Oswaldo Cruz; resgate; ciência; história; Brasil.

\section{Abstract}

One of the oldest, most varied and widest-ranging entomology collections in Latin America, containing a significant portion of Brazil's biodiversity, was severely damaged during an episode which came to be known as the Manguinhos Massacre, when ten eminent researchers had their political rights suspended. This note tells of how a particular episode in the tragedy caused part of this collection to be broken up, and how 8,554 valuable specimens of the order Diptera, from 35 families, including 99 type specimens, have been reintroduced to Instituto Oswaldo Cruz's Entomology Collection in their original positions in the steel cabinets 35 years on. This reintroduction represents the first effort to restore this collection both historically and scientifically since Brazil's military dictatorship.

Keywords: Entomology collection; Instituto Oswaldo Cruz; restoration; science; history; Brazil. 
Jane Costa et al.

A Coleção Entomológica do Instituto Oswaldo Cruz, uma das mais ricas coleções da América Latina, inclui insetos de todas as ordens. Esse acervo teve início em 1901, a partir da descrição de Anopheles lutzi, mosquito descrito pelo próprio Oswaldo Cruz, que foi a primeira espécie nova proposta em Manguinhos. O nome específico foi dado em homenagem a Adolpho Lutz, que além de pesquisador de grande projeção e reconhecimento no mundo científico, era também amigo de Oswaldo Cruz (Benchimol, Sá, 2005).

A Coleção Entomológica teve grande impulso como conseqüência das expedições realizadas pelos pesquisadores no início do século XX. Nesse contexto seguiram-se as descrições de inúmeras espécies e gêneros novos realizadas por outros renomados cientistas da época, tais como Adolpho Lutz, Arthur Neiva, Carlos Chagas, César Pinto, Costa Lima, Fábio Leoni Werneck, Lauro Travassos e Otávio Mangabeira Filho, que colecionaram e trabalharam em grupos específicos em seus laboratórios, enriquecendo a Coleção. Em 1909 editou-se o primeiro catálogo de espécies, sendo esta a primeira publicação do Instituto Oswaldo Cruz. Na listagem das coleções constam: 95 mosquitos, 145 tabanídeos e 40 carrapatos, além de uma coleção de helmintos (Figura 1).

Atualmente, o acervo da Coleção Entomológica do Instituto Oswaldo Cruz está estimado em cerca de cinco milhões de insetos, preservados a seco em alfinetes de aço dispostos em gavetas de madeira com tampa de vidro (Figura 2), em lâminas montadas com bálsamo do Canadá, ou mantidos em álcool 70\% (Figura 3). Estão acondicionados em 19 armários de madeira e 87 armários de aço, em cinco salas do segundo andar do Pavilhão Mourisco.

Em 1970, durante o regime militar, ocorreu o episódio que ficou conhecido como Massacre de Manguinhos, termo cunhado por Lent (1972). Esse fato marcou catastroficamente a história do Instituto Oswaldo Cruz, pois dez de seus pesquisadores foram cassados, entre eles os entomologistas Herman Lent, Hugo de Souza Lopes e Sebastião José de Oliveira (Lent, 1978). O 'massacre' não se limitou apenas à expulsão de renomados cientistas da instituição; toda a estrutura física que havia foi integralmente desmantelada, e os armários contendo material científico foram transportados em condições inadequadas para o porão do antigo prédio do Hospital Evandro Chagas, no campus de Manguinhos, ocasionando perdas e danos irreparáveis a inúmeros exemplares da coleção. Naquele momento, algumas partes do acervo foram enviadas para outras instituições com o intuito de protegê-las e também para darem suporte a projetos de pesquisa em andamento. Com esse objetivo, o Museu de Zoologia da Universidade de São Paulo (MZUSP) recebeu dípteros da Coleção Entomológica do IOC, que ficaram sob a responsabilidade dos pesquisadores Nelson Papavero (MZUSP) e Ângelo Pires do Prado (Unicamp).

Em 1977 uma grande parte do acervo que havia sido depositado no Hospital Evandro Chagas retornou ao segundo andar do Pavilhão Mourisco, graças ao empenho de José Jurberg e o apoio do técnico de laboratório Orlando Vicente Ferreira. Este tinha sido assistente do mais eminente dos entomólogos brasileiros, Ângelo Moreira da Costa Lima, que iniciou sua carreira em Manguinhos em 1913. Em 1986, com a reintegração dos pesquisadores cassados, Sebastião José de Oliveira foi convidado a assumir a chefia e a curadoria da Coleção Entomológica, cargo que ocupou até seu falecimento, em abril de 2005, aos 87 anos de idade. Durante esse período, dedicou-se principalmente ao estudo da família Chironomidae. 


\section{Malleina.}

Preparada pelo processo habitual de Nocard. E' fornecida em

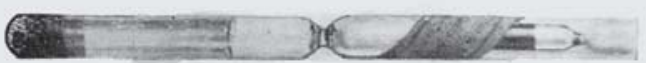

pequenos tubos fechados na parte media. Em um dos la-

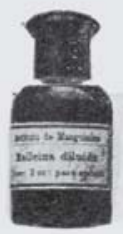
dos existe em pequena empoula a quantidade de mallei-

na bruta, necessaria para a inoculação de um cavallo. Do outro lado, a quantidade de agua physiologica esterilizada necessaria para o preparo da solução a inocular.

\section{Culturas e Collecções.}

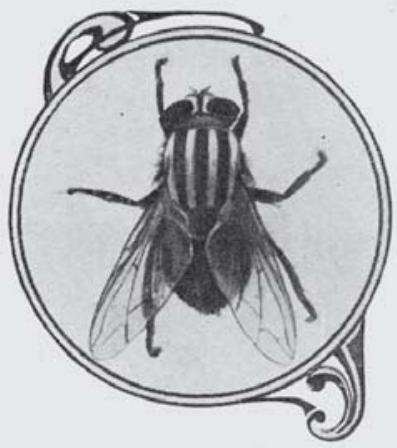

O Instituto possue colleccão de cerca de 500 amostras, em culturas puras, de bacterias pathogenas, saprophytas, cogumellos, levedos, etc., parte isoladas no proprio Instituto, e parte obtidas de outros

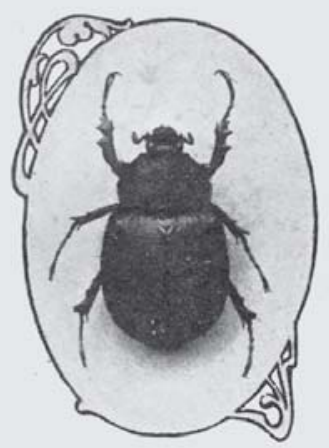

estabelecimentos. Possue o museu, peças anatomicas das principaes molestias tropicaes, como febre-amarella, peste, ankylostomiase, dysenteria, beri-beri, etc. Existe mais 'colleç̧ão de mosquitos brazileiros, cons-

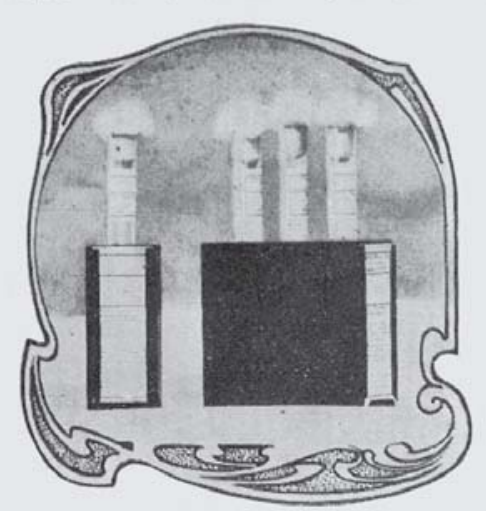
tituida de exemplares adultos, larvas, nymphas, desenhos elucidativos differentes, e preparados microscopios. Ainda ha ricas colle-

cções de tabánidas (mutucas) ixódidas (carrapatos), alem de outros animaes sugadores pertencentes aos dipteros (borrachudos maruis) hemipteros (barbeiros, perceveịos, etc.), aphanipteros (pulgas), etc.

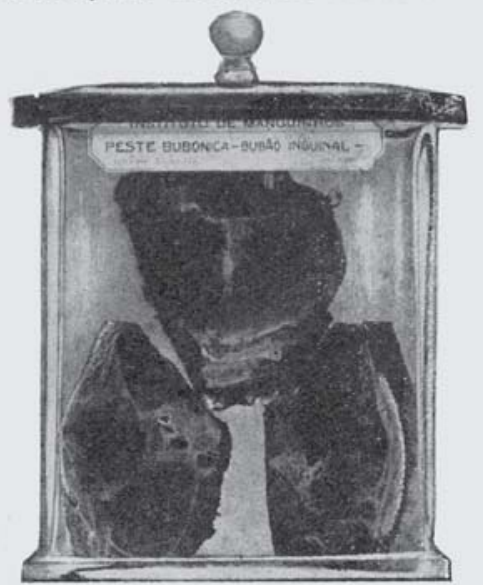

Figura 1 - Página da primeira publicação do Instituto Oswaldo Cruz, de 1909, onde as coleções entomológicas são citadas (exemplar da Biblioteca de Obras Raras de Manguinhos) 


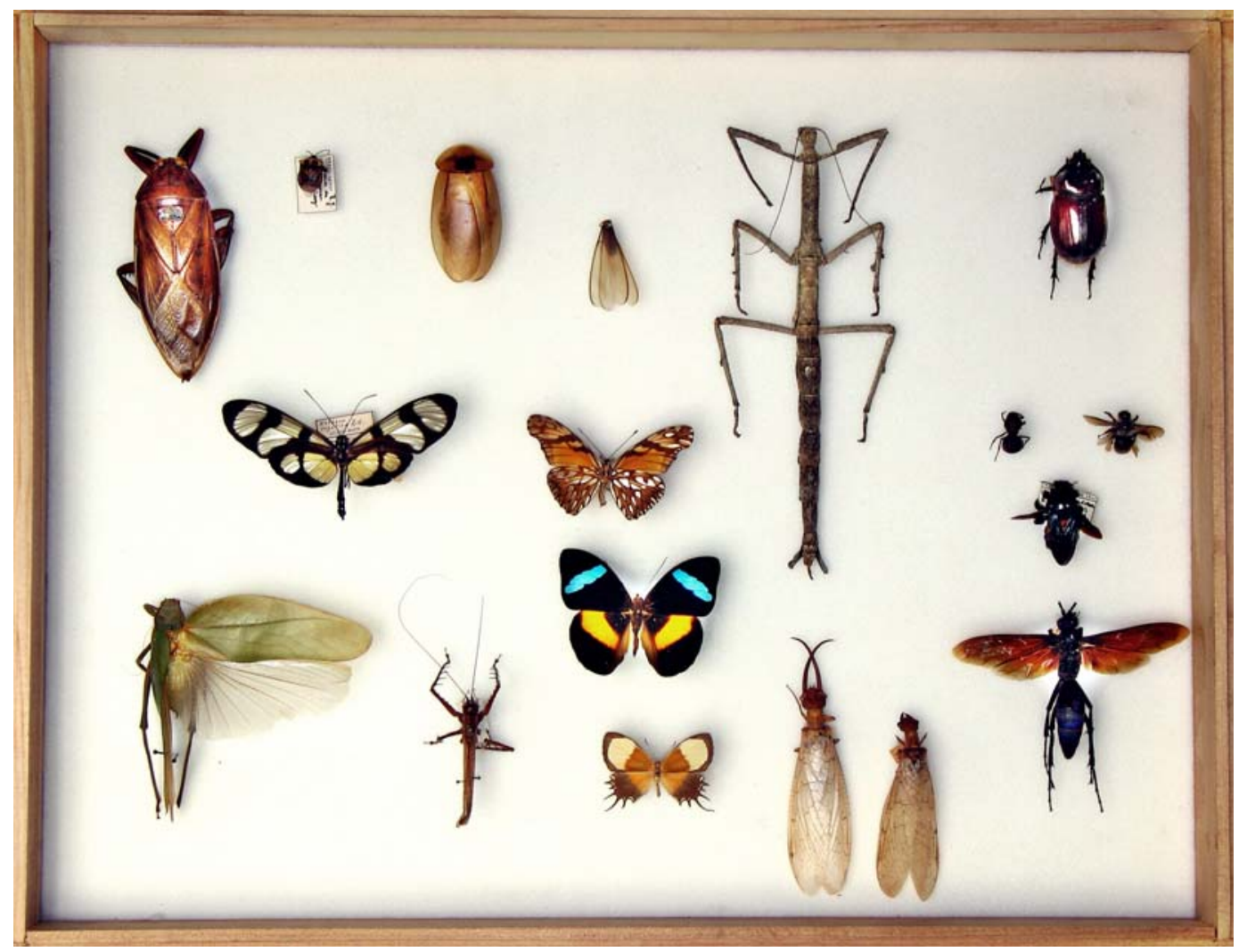

Figura 2 - Aspecto geral da gaveta de madeira utilizada na Coleção Entomológica do Instituto Oswaldo Cruz

Apenas em outubro de 2005, após 35 anos do Massacre de Manguinhos, graças ao esforço conjunto da Diretoria do IOC, da chefe do Departamento de Entomologia, Elizabeth Rangel, e da interação entre o curador da Coleção de Diptera do MZUSP e a curadora da Coleção Entomológica do Instituto Oswaldo Cruz, o valioso material que havia sido enviado ao Museu retornou e foi reincorporado ao acervo original. Ele encontra-se organizado em 51 gavetas, contendo um total de 8.554 exemplares distribuídos em 35 famílias, entre os quais se incluem 99 tipos, exemplares importantes e valiosos, pois nestes são baseadas as descrições de espécies, e seu exame permite elucidar uma série de questões taxonômicas (Martins, 1994). As gavetas foram novamente alocadas nos armários de aço da Coleção Entomológica nas suas posições originais, graças às etiquetas que se mantiveram ao longo desses anos (Figura 4).

Essa reincorporação representa o primeiro resgate de material após o Massacre de Manguinhos, o que é institucionalmente importante não só no contexto histórico, mas também científico, já que muitos dos exemplares retornados serviram como modelos para a descrição de espécies novas, além de terem sido coletados e/ou identificados por renomados entomólogos, tais como: Adolpho Lutz, Ângelo Pires do Prado, Hugo de Souza Lopes, Joseph Francisco Zikán, Moacir Alvarenga, Nelson Papavero, entre outros, incluindo o eminente geneticista Theodosius Dobzhansky, que realizou capturas entomológicas quando veio ao Brasil para conferir um curso sobre mecanismos de evolução das espécies na 
Faculdade de Filosofia Ciências e Letras da Universidade de São Paulo (Dobzhansky, 1944), além de acrescentar à coleção várias espécies com importância médica, veterinária e agrícola.

O conteúdo do acervo, reincorporado, organizado e quantificado em número de exemplares por famílias de Diptera, encontra-se na Tabela 1.

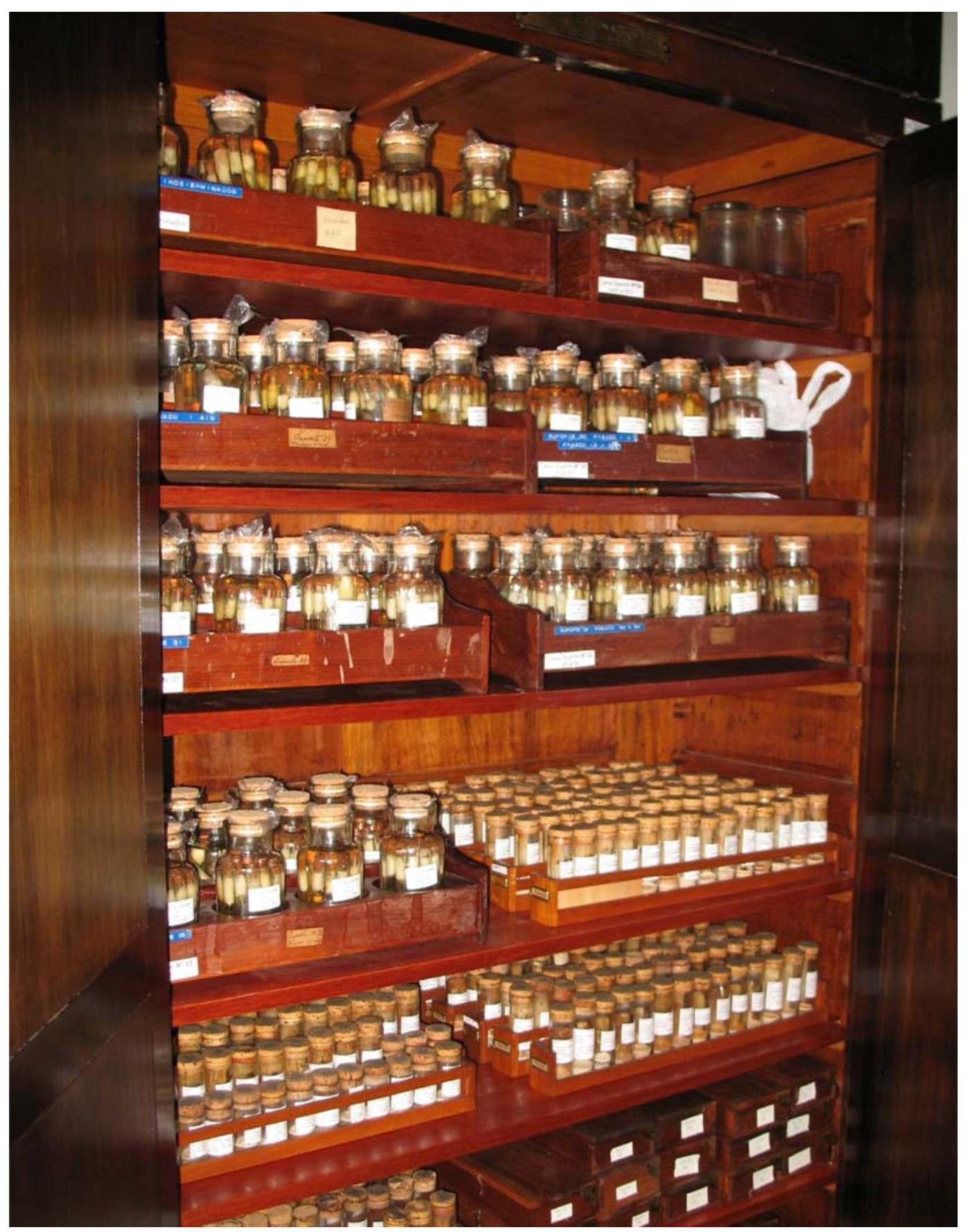

Figura 3 - Armário de madeira com os frascos nos quais os insetos ficam conservados em álcool 70\% 


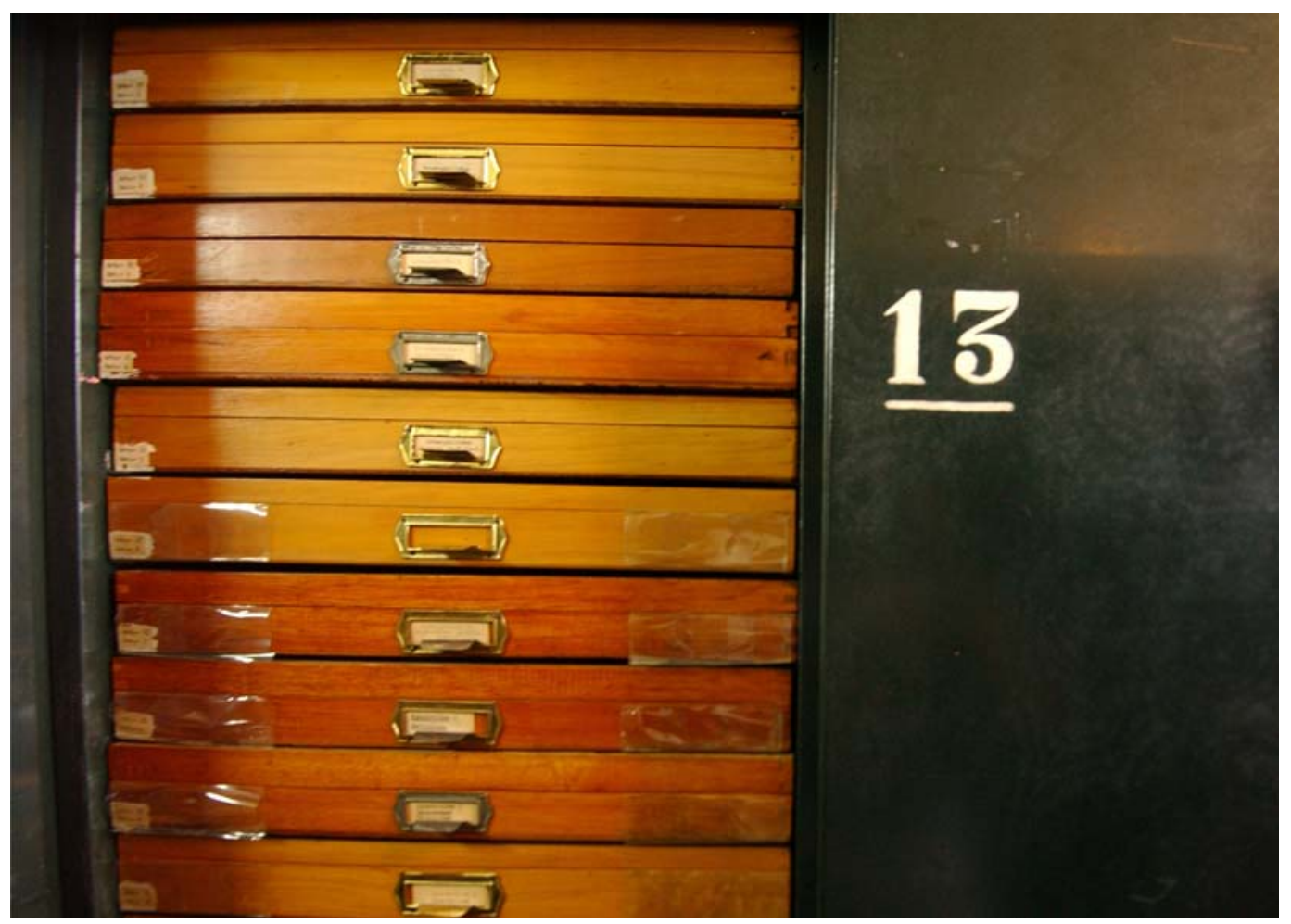

Figura 4 - Detalhe dos armários de aço e das etiquetas das gavetas que permitiram a reincorporação do material em seus sítios originais, na Coleção Entomológica do Instituto Oswaldo Cruz

Tabela 1 - Dípteros reincorporados à Coleção Entomológica do Instituto Oswaldo Cruz

\begin{tabular}{lc}
\hline Famílias & Número de exemplares \\
\hline Agromyzidae & 447 \\
Asteidae & 1 \\
Coelopsidae/Chyromyidae/Helomyzidae/Trixoscelidae & 14 \\
Canacaeidae & 39 \\
Carniidae/Chryptochetidae/Milichidae/Odinidae & 236 \\
Drosophilidae & 1116 \\
Dryomyzidae & 4 \\
Ephydridae & 1861 \\
Lauxaniidae & 139 \\
Leptoceratidae/Sphaeroceridae & 14 \\
Lonchaeidae/Pallopteridae/Tachiniscidae & 127 \\
Micropezidae & 353 \\
Neriidae & 295 \\
Otitidae & 16 \\
Periscelididae & 2 \\
Pterocallidae & 219 \\
Platistomatidae & 214 \\
Piophilidae/Sepsidae & 118 \\
Pyrgotidae & 105 \\
Ropalomeridae & 777 \\
Sciomyzidae & 128 \\
Tanypezidae & 77 \\
Tethinidae & 274 \\
Trypetidae & 735 \\
Tylidae & 413 \\
Ulididae & 400 \\
Sem identificação & 430 \\
Total & $\mathbf{8 5 4}$ \\
\hline
\end{tabular}


Infelizmente, muito do material danificado e ou extraviado durante o calamitoso Massacre de Manguinhos não poderá ser recuperado, sendo igualmente lamentáveis os efeitos do tempo perdido que jamais poderão ser minimizados. Entretanto, atualmente, significativos esforços institucionais têm sido realizados para a projeção, divulgação e modernização deste valioso acervo que alberga não só uma relevante parcela de nossa rica biodiversidade, mas também importantes depoimentos de nossa história científica e cultural. Esse acervo tem vida própria e continua a crescer em decorrência das atividades de inúmeros pesquisadores dedicados à entomologia e, principalmente, às doenças infecciosas. A relevância abrangente da Coleção Entomológica estrutura-se nas suas instigantes multifacetas que incentivam a mescla entre ciência, saúde, educação e história e, ainda que desnecessário fosse, conquista e arrebata através de sua incrível beleza estética.

\section{Tipos reincorporados}

Agromizidae

Haplomyza lopezi Oliveira \& Silva, 1954

Manguinhos RJ - Brasil, Oliveira, 1952 (7 exemplares).

Drosophilidae

Drosophila addisoni Pavan

Belém, PA - Brasil, 1948.

Drosophila andina Dobzhansky \& Pavan, 1943

Huancaya - Peru, 1943 (2 exemplares).

Drosophila ararama Pavan \& Cunha, 1947

Bertioga, SP - Brasil

Drosophila arauna Pavan \& Nacrur, 1950

Pirassununga, SP - Brasil

Drosophila austrorepleta Dobzhansky \& Pavan, 1943

Mogi das Cruzes, SP - Brasil, 1943 (2 exemplares).

Drosophila betari Dobzhansky \& Pavan, 1943

Iporanga, SP - Brasil, Iporanga, 1943 (2 exemplares).

Drosophila bocainensis Carson, 1954

Vila Atlântica - Brasil, Carson, 1951 (2 exemplares).

Drosophila bandeirantorum Dobzhansky \& Pavan, 1943

Mogi das Cruzes, SP - Brasil, 1943 (2 exemplares).

Drosophila brunneipalpa Dobzhansky \& Pavan, 1943

Apiaí, SP - Brasil, 1943 (2 exemplares).

Drosophila camargoi Dobzhansky \& Pavan, 1943

Belém, PA - Brasil, 1942 (2 exemplares). 
Jane Costa et al.

Drosophila cardinoides Dobzhansky \& Pavan, 1943

Iporanga, SP - Brasil, 1943 (2 exemplares).

Drosophila capricorni Dobzhansky \& Pavan, 1943

Praia Grande, SP - Brasil, 1943 (2 exemplares).

Drosophila dreyfusi Dobzhansky \& Pavan, 1943

Mogi das Cruzes, SP - Brasil, 1943.

Drosophila fasciocoloides Dobzhansky \& Pavan, 1943

Bertioga, SP - Brasil, 1943.

Drosophila guaramuru Dobzhansky \& Pavan, 1943

Amália, SP - Brasil, 1941 (2 exemplares).

Drosophila guarani Dobzhansky \& Pavan, 1943

Cantareira, SP - Brasil, 1943 (2 exemplares).

Drosophila guaru Dobzhansky \& Pavan, 1943

Amália, SP - Brasil, G. Sebreiser (2 exemplares).

Drosophila inca Dobzhansky \& Pavan, 1943

Huancuyo - Perú, 1943 (2 exemplares).

Drosophila krungii Pavan \& Breuer

Cantareira, SP - Brasil, 1924.

Drosophila mediopunctata Dobzhansky \& Pavan, 1943

Itanhaem, SP - Brasil, 1943 (2 exemplares).

Drosophila mediosignata Dobzhasnky \& Pavan, 1943

Iporanga, SP - Brasil, 1943 (2 exemplares).

Drophila mirim Dobzhansky \& Pavan, 1943

Bertioga, SP - Brasil, 1943 (2 exemplares).

Drosophila moju Pavan, 1950

Belém, PA - Brasil, 1948.

Drosophila neolliptica Pavan \& Magalhães

Anápolis, GO - Brasil, 1949 (2 exemplares).

Drosophila neosaltans Pavan \& Magalhães, 1950

Mogi das Cruzes, SP - Brasil, 1948.

Mogi das Cruzes, SP - Brasil, 1949.

Drosophila novemaristata Dobzhansky \& Pavan, 1943

Huancaya - Peru, 1943 (2 exemplares).

Drosophila onca Dobzhansky \& Pavan, 1943

Iporanga, SP - Brasil, 1943. 
Drosophila pallidipennis Dobzhansky \& Pavan, 1943

Iporanga, SP - Brasil, 1943 (2 exemplares).

Drosophila para Pavan \& Burla, 1950

Belém, PA - Brasil, 1948 (2 exemplares).

Drosophila parabocainoides Wheeler \& Magalhães, 1962

Feliz, RS - Brasil, Carson, 1951 (2 exemplares).

Drosophila pararepleta Dobzhansky \& Pavan, 1943

Mogi das cruzes, SP - Brasil, 1943 (2 exemplares).

Drosophila paulista Dobzhansky \& Pavan, 1943

Campinas, SP - Brasil, 1943 (2 exemplares).

Drosophila polymorpha Dobzhansky \& Pavan, 1943

Bertioga, SP - Brasil, 1943 (2 exemplares).

Drosophila setosa Dobzhansky \& Pavan, 1943

Mogi das Cruzes, SP - Brasil, 1943 (2 exemplares).

Drosophila tuchaua Pavan, 1950

Belém, PA - Brasil, 1948 (2 exemplares).

Rhinoleucophanga angustifrons Malog., 1946

Angra dos Reis, RJ - Brasil, Travassos \& Lopes, 1934.

Rhinoleucophanga lopesi Malog., 1946

RJ - Brasil, Lopes, 1934.

Rhinoleucophanga matogrossensis Malog., 1946

Salobra, MT - Brasil, Com. IOC, 1940.

Rhinoleucophanga personata Malog., 1946

Salobra, MT - Brasil, Com. IOC, 1941 (2 exemplares).

Ephidridae

Dimecoenia grumani Oliveira, 1954

Torres, RS - Brasil, E. Gruman, 1950 (2 exemplares).

Dimecoenia lenti Oliveira, 1954

Província de Antofagasta - Chile, H. Lent, 1952.

Dimecoenia lopesi Oliveira, 1954

Recreio dos Bandeirantes, RJ - Brasil, Lopes, 1940.

Dimecoenia venteli Oliveira, 1954

Bodoquena, MS - Brasil, Com. IOC, 1941.

Ephydrella freitasi Oliveira, 1954

Bodoquena, MS - Brasil, Com. IOC, 1941. 
Jane Costa et al.

\section{Micropezidae}

Protylos littoralis

Barra da Tijuca, RJ - Brasil, Lopes, 1965 (2 exemplares).

Protylos reconditus

Tijuca, RJ - Brasil, Lopes, 1965 (3 exemplares).

Grajaú, RJ - Brasil, Lopes, 1965.

RJ - Brasil, Lopes, 1933.

Palmeiras, RJ - Brasil, Lopes, 1934.

São Conrado, RJ - Brasil, Lopes, 1965.

Protylos stigmaticus W. D.

Belém, PA - Brasil, Lopes, 1965 (9 exemplares).

\section{AGRADECIMENTOS}

Ao doutor Márcio Felix, pela revisão do manuscrito.

\section{REFERÊNCIAS BIBLIOGRÁFICAS}

BENCHIMOL, Jaime Larry; SÁ, Magali Romero de. Adolpho Lutz: obra completa. v.2, livro 1. Rio de Janeiro: Editora Fiocruz. 2005.

DOBZHANSKY, Theodosius.

Mecanismo da evolução e origem das espécies. Rio Janeiro: Centro Nacional de Ensino e Pesquisas Agronômicas/Ministério da Agricultura.

(Boletim do Cursos de Aperfeiçoamento e Especialização, 2). 1944.

LENT, Herman.

O massacre de Manguinhos. Rio de Janeiro: Editora Avenir. 1978.
LENT, Herman.

Desenvolvimento da entomologia no Instituto Oswaldo Cruz: discurso na Academia Brasileira de Ciências ao receber o Prêmio Costa Lima. Ciência e Cultura, São Paulo, v.24, n.12, p.1192-1200. 1972.

MARTINS, Ubirajara Ribeiro.

A coleção taxonômica. In: Papavero, N. (Org.). Fundamentos práticos da taxonomia zoológica (coleções, bibliografia, nomenclatura). São Paulo: Universidade Estadual Paulista. p.19-43. 1994. 